\title{
Electron correlations in narrow energy bands: modified polar model approach*
}

\author{
L.Didukh† Yu.Skorenkyy, O.Kramar \\ Ternopil State Technical University, Department of Physics, 56 Rus'ka Str., 46001 Ternopil, Ukraine
}

Received April 29, 2008, in final form July 2, 2008

\begin{abstract}
The electron correlations in narrow energy bands are examined within the framework of the modified form of polar model. This model permits to analyze the effect of strong Coulomb correlation, inter-atomic exchange and correlated hopping of electrons and explain some peculiarities of the properties of narrow-band materials, namely the metal-insulator transition with an increase of temperature, nonlinear concentration dependence of Curie temperature and peculiarities of transport properties of electronic subsystem. Using a variant of generalized Hartree-Fock approximation, the single-electron Green's function and quasi-particle energy spectrum of the model are calculated. Metal-insulator transition with the change of temperature is investigated in a system with correlated hopping. Processes of ferromagnetic ordering stabilization in the system with various forms of electronic DOS are studied. The static conductivity and effective spin-dependent masses of current carriers are calculated as a function of electron concentration at various DOS forms. The correlated hopping is shown to cause the electron-hole asymmetry of transport and ferromagnetic properties of narrow band materials.
\end{abstract}

Key words: polar model of crystal, Mott-Hubbard material, ferromagnetic ordering, conductivity

PACS: $71.10 . F d, 71.27 .+a, 72.20 .-i, 75.10 .-b$

\section{Introduction}

Polar model of crystal, developed on the basis of pioneering work of Schubin and Wonsowsky [1], proved to have rich physical content. Within the framework of the polar model the existence of systems with charge ordering and gapless semiconductors was predicted, the criterium for metalinsulator transition was formulated for the first time, the explanation of fractional magnetic moment of transition $3 d$-metals was proposed, the possibility of indirect (through polar states) exchange interaction was shown. In fact, Hubbard model [2], which today is a standard model for describing strong electron correlations in crystals, is a partial case of the polar model. An idea of the configurational representation [3] (in a polar model, site "configurations" are homeopolar states, holes and doublons) appeared to be very fruitful in investigations of a wide class of materials with unique electrical and magnetic properties, namely oxides, sulphides and selenides of transition and rare-earth elements. However, direct application of the method proposed in work [1] in many cases was found to be inefficient. It was due to, firstly, the difficulties of constructing Hamiltonians of each particular system, because the algorithm of transition from electron representation to configurational one had not been devised, such a transition even in the simplest model of $s$-band was too cumbersome. The second reason of inefficiency of early forms of a polar model is uncontrolled character of approximations used, especially the postulating of commutation relations (of bose-type) for operators of site excitations and replacement of some operators with $c$-numbers (this way the Hamiltonian of "gas limit" was constructed). The other deficiency was the constraint of equality of the mean electron number to unity, what restricted the use of the model to the doped Mott-Hubbard insulators. Thus, in spite of the extraordinary heuristic value of a polar model, the mathematical foundation of configurational representation was not developed. It caused also the absence of effective mathematical methods of model Hamiltonians (in

\footnotetext{
${ }^{*}$ This article is dedicated to Prof. I.V. Stasyuk on the occasion of his 70th birthday.
}

†E-mail: didukh@tu.edu.te.ua 
configurational representation) treatment within the framework of the traditional form of a polar model.

The disadvantages mentioned above were partially removed in a "new form of polar model" of Glauberman, Vladimirov and Stasyuk [4-6], where a rigorous algorithm of the transition from electron operators to the so-called "site elementary excitation" operators was elaborated. For the latter, exact commutation relations of two types, namely quasifermi and quasibose, were established. However, the form of a polar model, presented in the works [4-6], is disadvantageous, namely, operators of site excitation were built over some postulated magnetic "background" and, as a result, the approach proposed in the mentioned papers is applicable only to ferromagnetic insulators.

The papers [7] and [8,9] were devoted to clarification of mathematical essence of configurational representation of a polar model. In the first paper, transition operators $X_{i}^{k l}$ (Hubbard operators) were introduced and in papers $[8,9]$ the relation between electron and configuration representations:

$$
a_{i s}=X_{i}^{0 s}-\eta_{s} X_{i}^{\bar{s} 2}
$$

was introduced. In the above equation, $a_{i s}$ is an operator of annihilation of electron with spin $s=\uparrow$ or $\downarrow$ at site $i, X_{i}^{k l}$ is an operator of site $i$ transition from state $|l\rangle$ to state $|k\rangle$, possible states are hole $|0\rangle$, doublon $|2\rangle$ and single occupied states with electron $|\uparrow\rangle$ or $|\downarrow\rangle, \eta_{s}=1$ for $s=\uparrow$ and $\eta_{s}=-1$ otherwise (in paper [9] other notations were used, here we use standard ones). The transition operator $X_{i}^{k l}$ is nothing but a product of respective Shubin-Wonsowsky operators: $X_{i}^{\gamma \delta}=\alpha_{i \gamma}^{+} \alpha_{i \delta}$ (see [10] in this connection). Commutation relations for a pair of Shubin-Wonsowsky operators are identical to the corresponding Hubbard operators. This fact closes the discussion on the statistics of Shubin-Wonsowsky operators. It should be noted that the algebra for ShubinWonsowsky operators as well as for operators introduced later in the papers [11,12], is "richer" than Hubbard operators' algebra, and the former operators can have both fermi and bose character (see, for example, work [13]).

Despite its bulky appearance, configurational representation of the polar model (or Hubbard model) for systems with strong intra-atomic Coulomb interaction essentially simplifies the analysis due to a diagonal form of the principal term, namely intra-atomic Coulomb interaction.

A series of works were devoted to mathematical treatment of polar model Hamiltonian by different methods (see work [14] for a review). Here we note the papers [10,15-22] in the context of further investigations concerning correlation effects in narrow-band materials. Within the framework of this approach, the efficient form of perturbation theory $[8,9]$ (generally adopted today) was developed, an effective Hamiltonian was formulated, and generalized $t-J$ model was proposed.

In the present paper specific electric and magnetic properties of some narrow-band materials are investigated within the framework of modified form of a polar model. In section 2, the model Hamiltonian is formulated. In section 3 the metal-insulator transition in a half-filled band with an increase of temperature is studied. In section 4 ferromagnetic ordering in a partially filled band is considered and nonlinear concentration dependence of Curie temperature is explained. In section 5 the peculiarities of transport properties of an electronic subsystem are analyzed.

\section{Modified form of polar model}

In electronic operator notations, the modified form of the polar model is represented by Hamiltonian $[10]$

$$
\begin{aligned}
H= & -\mu \sum_{i s} a_{i s}^{+} a_{i s}+\sum_{i j s}^{\prime} t_{i j}(n) a_{i s}^{+} a_{j s}+\sum_{i j s}^{\prime}\left(T(i j) a_{i s}^{+} a_{j s} n_{i \bar{s}}+h . c .\right) \\
& +U \sum_{i} n_{i \uparrow} n_{i \downarrow}+\frac{1}{2} \sum_{i j s s^{\prime}}^{\prime} J(i j) a_{i s}^{+} a_{j s^{\prime}}^{+} a_{i s^{\prime}} a_{j s}+\frac{1}{2} \sum_{i j s s^{\prime}}^{\prime} V(i j) n_{i s} n_{j s^{\prime}},
\end{aligned}
$$

where $\mu$ is chemical potential,

$$
t_{i j}(n)=t(i j)+n \sum_{\substack{k \neq i \\ k \neq j}} J(i k j k)
$$


is hopping integral between the nearest neighbors dependent on electron concentration $n$,

$$
J(i j k l)=\iint \phi^{*}\left(\mathbf{r}-\mathbf{R}_{i}\right) \phi\left(\mathbf{r}-\mathbf{R}_{k}\right) \frac{e^{2}}{\left|\mathbf{r}-\mathbf{r}^{\prime}\right|} \phi^{*}\left(\mathbf{r}^{\prime}-\mathbf{R}_{j}\right) \phi\left(\mathbf{r}^{\prime}-\mathbf{R}_{l}\right) \mathrm{d} \mathbf{r} \mathrm{d} \mathbf{r}^{\prime},
$$

are the matrix elements which describe electron-electron interactions, $U=J($ iiii $)$ is energy of intra-atomic Coulomb repulsion, $V(i j)=J(i j i j)$ describes inter-atomic Coulomb interaction $(i$ and $j$ are the nearest neighbors $), T(i j)=J(i i i j)$ describes the effect of site occupation on the hopping processes (correlated hopping), $J(i j)=J(i j j i)$ is inter-atomic ferromagnetic exchange interaction. Having neglected all matrix elements in (2) except $t(i j)$ and $U$ we obtain the Hubbard Hamiltonian.

We rewrite the Hamiltonian of a correlated electron system in representation of $X_{i}^{k l}$ Hubbard operators using relations (1):

$$
\begin{gathered}
H=H_{0}+H_{\mathrm{tr}}+H_{\mathrm{tr}}^{\prime}+H_{\mathrm{ex}}, \\
H_{0}=-\mu \sum_{i s}\left(X_{i}^{s}+X_{i}^{2}\right)+U \sum_{i} X_{i}^{2}+\frac{1}{2} \sum_{i j}^{\prime} V(i j)\left(1-X_{i}^{0}+X_{i}^{2}\right)\left(1-X_{j}^{0}+X_{j}^{2}\right), \\
H_{\mathrm{tr}}=\sum_{i j s}^{\prime} t_{i j}(n) X_{i}^{s 0} X_{j}^{0 s}+\sum_{i j s}^{\prime} \tilde{t}_{i j}(n) X_{i}^{2 s} X_{j}^{s 2}, \\
H_{\mathrm{tr}}^{\prime}=\sum_{i j s}^{\prime}\left(t_{i j}^{\prime}(n) \eta_{s} X_{i}^{s 0} X_{j}^{\bar{s} 2}+h . c .\right), \\
H_{\mathrm{ex}}=-\frac{1}{2} \sum_{i j s}^{\prime} J(i j)\left(\left(X_{i}^{s}+X_{i}^{2}\right)\left(X_{j}^{s}+X_{j}^{2}\right)+X_{i}^{s \bar{s}} X_{j}^{\bar{s} s}\right) .
\end{gathered}
$$

Here translation processes of holes and doublons are characterized by different hopping integrals, $t_{i j}(n)=\left(1-\tau_{1} n\right) t_{i j}$ and $\tilde{t}_{i j}(n)=\left(1-\tau_{1} n-2 \tau_{2}\right) t_{i j}$, respectively; $t_{i j}^{\prime}(n)=\left(1-\tau_{1} n-\tau_{2}\right) t_{i j}$ is a hopping parameter, which describes processes of creation and destruction of doublon-hole pair; correlated hopping parameters $\tau_{2}$ and $\tau_{1}$ describe the effect of the sites involved the hopping process and neighboring sites, respectively.

Configurational representation is especially useful in investigating a narrow-band system in which the condition $U \gg w(2 w=2 z|t(i j)|$ is bare bandwidth, $z$ is a number of the nearest neighbors to a site) is satisfied. In this case, the system can be both Mott-Hubbard insulator at $n=1$ and doped Mott-Hubbard insulator at $n \neq 1$. Then, general Hamiltonian, using a suitable form of perturbation theory [8], can be written in the form of effective Hamiltonian (EH), which is suitable for the mathematical treatment. In this way the transition to the well-known $t-J$ model was done (see the review [15] as well as the papers [8,23], where the modern form of $t-J$ model was formulated for the first time). The canonical transformation is performed (see [10] for details) and the Hamiltonian (5) is written in the form

$$
\tilde{H}=H_{0}+H_{\text {tr }}+H_{\text {ex }}+\tilde{H}_{\mathrm{ex}}+\tilde{H}_{\text {tr-ex }},
$$

where

$$
\begin{aligned}
\tilde{H}_{\mathrm{ex}} & =-\frac{1}{2} \sum_{i j s}^{\prime} \tilde{J}(i j)\left(X_{i}^{s} X_{j}^{\bar{s}}-X_{i}^{s \bar{s}} X_{j}^{\bar{s} s}-X_{i}^{2} X_{j}^{0}\right) \\
\tilde{H}_{\mathrm{tr}-\mathrm{ex}} & =-\frac{1}{2} \sum_{i j k s}^{\prime} J(i j k)\left(X_{i}^{s 0} X_{j}^{\bar{s}} X_{k}^{0 s}-X_{i}^{s 0} X_{j}^{\bar{s} s} X_{k}^{0 \bar{s}}+X_{i}^{2 s} X_{j}^{s \bar{s}} X_{k}^{\bar{s} 2}-X_{i}^{2 s} X_{j}^{\bar{s}} X_{k}^{s 2}\right) .
\end{aligned}
$$

Here $\tilde{J}(i j)=2 t_{i j}^{\prime}(n) t_{i j}^{\prime}(n) / \Delta$ is integral of indirect exchange (through polar states), $J(i j k)=$ $2 t_{i j}^{\prime}(n) t_{j k}^{\prime}(n) / \Delta$ is integral of indirect charge transfer, $\Delta=U-V+z V\left(\left\langle X_{i}^{0}\right\rangle+\left\langle X_{i}^{2}\right\rangle\right)$. The distinctions of $\mathrm{EH}$ (6) from other forms of $t-J$ models [24,25] are caused by the concentration dependence of hopping integrals in lower and upper subbands, the difference of the noted hopping integrals (the absence of electron-hole symmetry) and unusual form of the concentration dependent superexchange and superhopping integrals. The peculiarities of the model EH listed above are useful in interpreting the physical properties of narrow-band materials. 


\section{Metal-insulator transition at increase of temperature}

Among the metal-insulator transitions (MIT) observed in narrow-bands materials the transitions from paramagnetic metal state to paramagnetic insulator state at increase of temperature are of special interest. Such transitions are realized in systems $\mathrm{NiS}_{2-x} \mathrm{Se}_{x}[26,27]$ and $\left(\mathrm{V}_{1-x} \mathrm{Cr}_{x}\right)_{2} \mathrm{O}_{3}[26$, 28 . In these systems, the paramagnetic insulator - paramagnetic metal transitions under external pressure are observed as well. In this section temperature-induced MIT is considered and explained based on the modified form of polar model.

Let us consider an electron system described by Hamiltonian (5) in the case of intermediate correlation strength $U \simeq w(n)$ and introduce a single-particle Green's function

$$
\left\langle\left\langle a_{p \uparrow} \mid a_{p^{\prime} \uparrow}^{+}\right\rangle\right\rangle=\left\langle\left\langle X_{p}^{0 \uparrow} \mid X_{p^{\prime}}^{\uparrow 0}\right\rangle\right\rangle-\left\langle\left\langle X_{p}^{\downarrow 2} \mid X_{p^{\prime}}^{\uparrow 0}\right\rangle\right\rangle-\left\langle\left\langle X_{p}^{0 \uparrow} \mid X_{p^{\prime}}^{2 \downarrow}\right\rangle\right\rangle+\left\langle\left\langle X_{p}^{\downarrow 2} \mid X_{p^{\prime}}^{2 \downarrow}\right\rangle\right\rangle .
$$

The functions $\left\langle\left\langle X_{p}^{0 \uparrow} \mid X_{p^{\prime}}^{\uparrow 0}\right\rangle\right\rangle$ and $\left\langle\left\langle X_{p}^{\downarrow 2} \mid X_{p^{\prime}}^{\uparrow 0}\right\rangle\right\rangle$ satisfy the equations of motion

$$
\begin{aligned}
(E+\mu)\left\langle\left\langle X_{p}^{0 \uparrow} \mid X_{p^{\prime}}^{\uparrow 0}\right\rangle\right\rangle & =\frac{\delta_{p p^{\prime}}}{2 \pi}\left\langle X_{p}^{\uparrow}+X_{p}^{0}\right\rangle+\left\langle\left\langle\left[X_{p}^{0 \uparrow}, H_{\mathrm{tr}}\right] \mid X_{p^{\prime}}^{\uparrow 0}\right\rangle\right\rangle+\left\langle\left\langle\left[X_{p}^{0 \uparrow}, H_{\mathrm{tr}}^{\prime}\right] \mid X_{p^{\prime}}^{\uparrow 0}\right\rangle\right\rangle, \\
(E+\mu-U)\left\langle\left\langle X_{p}^{\downarrow 2} \mid X_{p^{\prime}}^{\uparrow 0}\right\rangle\right\rangle & =\left\langle\left\langle\left[X_{p}^{\downarrow 2}, H_{\mathrm{tr}}\right] \mid X_{p^{\prime}}^{\uparrow 0}\right\rangle\right\rangle+\left\langle\left\langle\left[X_{p}^{\downarrow 2}, H_{\mathrm{tr}}^{\prime}\right] \mid X_{p^{\prime}}^{\uparrow 0}\right\rangle\right\rangle,
\end{aligned}
$$

with $[A, B]=A B-B A$. To obtain the closed system of equations we take advantage of the two-pole approximation $[16,19]$. Suppose in equation $(8)$

$$
\left[X_{p}^{0 \uparrow}, H_{\mathrm{tr}}\right]=\sum_{j} \epsilon(p j) X_{j}^{0 \uparrow}, \quad\left[X_{p}^{\downarrow 2}, H_{\mathrm{tr}}\right]=\sum_{j} \tilde{\epsilon}(p j) X_{j}^{\downarrow 2}
$$

where $\epsilon(p j)$ and $\tilde{\epsilon}(p j)$ are non-operator quantities, which can be calculated using the method of work [10]. For half band filling (electron concentration $n=1$ ) in a paramagnetic state we have

$$
\begin{gathered}
\epsilon(p j)=\left(1-2 d+2 d^{2}\right) t_{p j}-2 d^{2} \tilde{t}_{p j}, \\
\tilde{\epsilon}(p j)=\left(1-2 d+2 d^{2}\right) \tilde{t}_{p j}-2 d^{2} t_{p j},
\end{gathered}
$$

with $d=\left\langle X_{p}^{2}\right\rangle$ being the concentration of the doubly occupied sites.

Terms of equations (8) describing processes of doublon-hole pair creation or destruction may be taken into account in the mean-field approximation. This way we obtain a closed system of equations. Applying the analogous procedure to equations for functions $\left\langle\left\langle X_{p}^{0 \uparrow} \mid X_{s}^{2 \downarrow}\right\rangle\right\rangle$ and $\left\langle\left\langle X_{p}^{\downarrow 2} \mid X_{s}^{2 \downarrow}\right\rangle\right\rangle$, after the Fourier transformation, we obtain Green's function

$$
G_{\mathbf{k}}=\frac{1}{2 \pi}\left(\frac{A_{\mathbf{k}}}{E-E_{1}(\mathbf{k})}+\frac{B_{\mathbf{k}}}{E-E_{2}(\mathbf{k})}\right), \quad A_{\mathbf{k}}=\frac{1}{2}\left(1-\frac{t^{\prime}(\mathbf{k})}{E_{2}(\mathbf{k})-E_{1}(\mathbf{k})}\right), \quad B_{\mathbf{k}}=1-A_{\mathbf{k}},
$$

where

$$
E_{1,2}(\mathbf{k})=-\mu+\frac{U}{2}+(1-2 d) \frac{t(\mathbf{k})+\tilde{t}(\mathbf{k})}{2} \mp \frac{1}{2} \sqrt{\left[U-(t(\mathbf{k})-\tilde{t}(\mathbf{k}))\left(1-2 d+4 d^{2}\right)\right]^{2}+\left(t^{\prime}(\mathbf{k})\right)^{2}}
$$

is the energy spectrum of the system, $t(\mathbf{k}), t^{\prime}(\mathbf{k})$ and $\tilde{t}(\mathbf{k})$ are Fourier transforms of hopping integrals $t_{i j}, t_{i j}^{\prime}$ and $\tilde{t}_{i j}$, respectively. Single-particle Green's function (12) and energy spectrum (13) are exact in the band and atomic limits and have peculiar dependence on concentration of polar states, which explains the temperature-induced transition to insulator state in the systems $\mathrm{NiS}_{2-x} \mathrm{Se}_{x}$ and $\left(\mathrm{V}_{1-x} \mathrm{Cr}_{x}\right)_{2} \mathrm{O}_{3}$. Equation (13) allows us to calculate the energy gap width as the energy difference between the bottom of the upper and the top of the lower Hubbard bands:

$$
\Delta E=-2 w(1-2 d)\left(1-\tau_{1}-\tau_{2}\right)+\frac{Q_{1}+Q_{2}}{2}
$$


where

$$
Q_{1,2}=\left(\left(U \mp 2 \tau_{2} C w\right)^{2}+\left(1-\tau_{1}-2 \tau_{2}\right)^{2} w^{2}\right)^{\frac{1}{2}}, \quad C=1-2 d+4 d^{2} .
$$

Expression (14) describes the closure of the energy gap in the spectrum of paramagnetic insulator at critical value $(U / w)_{\mathrm{c}}$, when the half-bandwidth $w$ increases (under pressure or doping). This expression also reproduces the exact result for a partial case of the model [29].

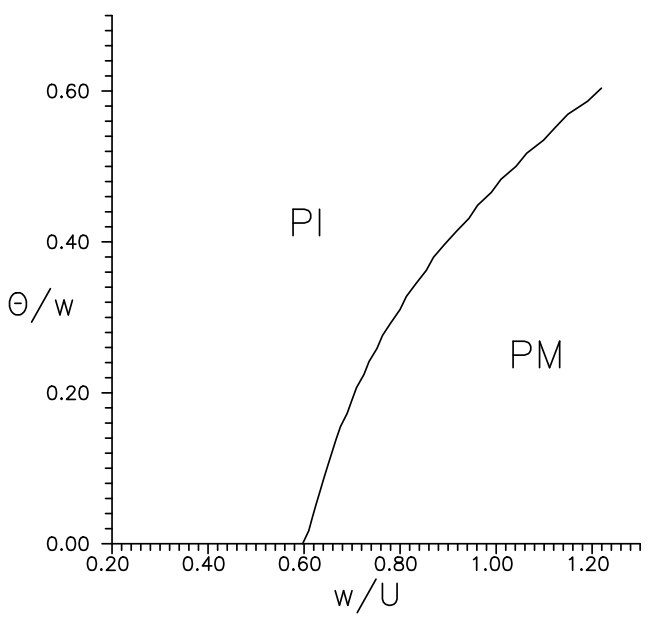

Figure 1. Phase diagram of paramagnetic metal - paramagnetic insulator transition.

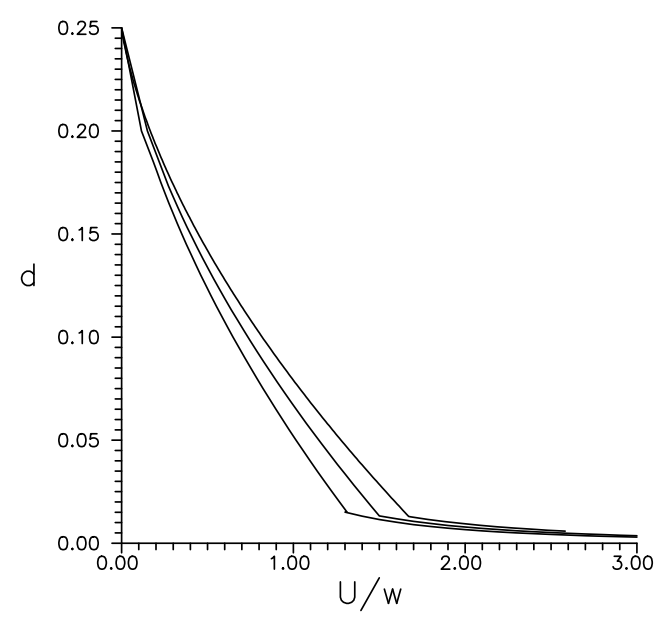

Figure 2. Polar states concentration as a function of the correlation strength parameter. Upper curve corresponds to $\tau_{2}=0$, middle curve: $\tau_{2}=0.1$, lower curve: $\tau_{2}=0.2$.

At increasing temperature in metallic state the overlapping of subbands decreases and temperature induced transition from metallic to insulating state can occur at some values of parameter $w / U$ (see figure 1). The energy gap depends on temperature through the concentration of polar states which can be derived from function $\left\langle\left\langle X_{p}^{\downarrow 2} \mid X_{s}^{2 \downarrow}\right\rangle\right\rangle$. The dependence $d(U / w)$ is plotted in figure 2. One can see that at critical value $(U / w)_{\mathrm{c}}$ the slope of $d(U / w)$-dependence changes; the concentration of doublons remains nonzero at any finite $U / w$. Our result for $d(U / w)$ at $\tau_{1}=\tau_{2}=0$ in the region of MIT is in good agreement with the results of the paper [30] obtained in the limit of infinite dimensions and composite operator method [31]. We have found that the correlated hopping processes reduce the polar states concentration and shift the transition point towards lower values of $U / w$. To the best of our knowledge, a systematic study of MIT in Hubbard model with correlated hopping, using the dynamical mean field theory, has not been performed so far, though an approach for the description of correlated hopping in infinite dimensions has been developed in the work [32]. Numerical data, obtained in the work [33] for a two-dimensional system with correlated hopping by Monte-Carlo calculations do not make it possible to study the temperature-induced MIT. Thus, the approximation developed in works $[10,16]$, is at the moment the most convenient tool for describing peculiar electric properties of transition-metal compounds with correlated hopping within the framework of the modified form of polar model.

\section{Ferromagnetic ordering in the model}

The regime of strong Coulomb interaction $(U \gg w)$ is more beneficial for ferromagnetic ordering realization in the considered model. In this case one may apply an effective Hamiltonian (6) obtained by the method of canonical transformation [10]. Energy spectrum of the system at $n<1$ is obtained in the form [34]:

$$
E_{s}(\mathbf{k})=-\mu+\alpha_{s} t_{\mathbf{k}}(n)+\beta_{s}-z J_{\mathrm{eff}} n_{s},
$$


here

$$
\alpha_{s}=1-n_{\bar{s}}+\frac{n_{\bar{s}} n_{s}}{1-n_{\bar{s}}}=\frac{2-n+\eta_{s} m}{2}+\frac{n^{2}-m^{2}}{2\left(2-n+\eta_{s} m\right)}
$$

is correlation narrowing factor,

$$
\beta_{s}=-\frac{1}{1-n_{\bar{s}}} \sum_{\mathbf{k}} t_{\mathbf{k}}(n)\left\langle X_{i}^{\bar{s} 0} X_{j}^{0 \bar{s}}\right\rangle_{\mathbf{k}}
$$

is spin-dependent shift of the subband center, $J_{\text {eff }}$ is effective exchange parameter, determined by competition of direct and indirect exchange interactions, $n_{s}=\left(n+\eta_{s} m\right) / 2, m$ is the system magnetization. For $n>1$ we obtain $\tilde{E}_{s}$ in a similar form; expressions for correlation narrowing factor and shift of subband center are obtained from equations (16) and (17) replacing $n \rightarrow 2-n$, $t(n) \rightarrow \tilde{t}(n)$. The analytical calculation of spin-dependent shift of the subband center $\beta_{s}$ for model rectangular density of states (DOS) has been done in the work [35]. In the present paper $\beta_{s}$ is calculated numerically and its value is shown to depend drastically on non-perturbed DOS form.

Let us calculate the ground state energy of the system described by effective Hamiltonian (6) in the case of $n<1$. We use the expression for the ground state energy (per lattice site) in the form $[36]$

$$
E_{0}=\frac{1}{2 N} \sum_{\mathbf{k} s} \int_{-\infty}^{\infty}\left[t_{\mathbf{k}}(n)+E\right] f(E) S_{\mathbf{k}}^{s}(E) \mathrm{d} E
$$

and concentration of electrons with spin $s$

$$
n_{s}=\frac{1}{N} \sum_{\mathbf{k}} \int_{-\infty}^{\infty} f(E) S_{\mathbf{k}}^{s}(E) \mathrm{d} E
$$

here $f(E)$ is the Fermi distribution function, $S_{\mathbf{k}}^{s}(E)=\left(1-n_{\bar{s}}\right) \delta\left(E-E_{\mathbf{k}}^{s}\right)$ is spectral density of the Green's function.

We argue that the form of non-interacting DOS (which corresponds to some lattice structure) substantially effects the critical electron concentration $n_{1}$ at which ferromagnetic ordering occurs as well as electron concentration $n_{2}$ at which magnetic moment becomes saturated. By numerical calculations of the ground state energy based on the expression (18) and subsequent minimization we have investigated the condition of ferromagnetism stabilization for various DOS. In particular, the numerical analysis has been done for DOS which corresponds to a simple cubic lattice [37], for DOS that corresponds to the body-centered cubic lattice [38], as well as for the DOS with the peak near the band-edge [39] $\rho(\epsilon)=c \sqrt{w^{2}-\epsilon^{2}} /(w+a \epsilon)$, with free parameter $c=\left(1+\sqrt{1-a^{2}}\right) /(\pi w)$. Varying the asymmetry parameter $a$, from the latter DOS one can obtain both the semi-elliptical DOS (at $a=0$ ) and the DOS with peak near the band-edge (at $a \rightarrow 1$ ).

Our results for sc-lattice $n_{1}=0.36$ and $n_{2}=0.62$ agree with the well-known results obtained by Roth [36]: at $n_{1}=0.36$ the ferromagnetic ordering occurs, and at $n_{2}=0.63$ the magnetic moment saturates. In the case of sc-lattice, spectral density approximation (SDA) [40,41] yields the following results: spontaneous magnetization occurs at $n_{1}=0.34$ and at $n_{2}=0.68$ ferromagnetic ordering reaches the saturation; in the case of bcc-lattice: the critical concentrations are $n_{1}=0.52$ and $n_{2}=0.68$, respectively. Our results for bcc-lattice are $n_{1}=0.55$ and $n_{2}=0.64$. The Gutzwiller variational approach [42] gives only the critical concentration of saturated ferromagnetic state $n_{2}=0.68$ for sc- and bcc-lattices. Our results also agree with the results obtained by the expansion of one-particle Green's functions over the inverse coordination number [43]. In the case of "tunable" DOS with the peak near the band-edge we have $n_{1}=0.20$ and $n_{2}=0.31$ at $a=0.3, n_{1}=0.09$ and $n_{2}=0.15$ at $a=0.15$. With an increase of parameter $a$, the critical concentration of ferromagnetism onset decreases. Therefore, the existence of a peak near the band-edge in electronic DOS favors the ferromagnetic ordering, in accordance with [39]. It should be noted, that in the case of strong 
electron correlation and half-filling of the band (when the shifts of subband center vanish) the ferromagnetic ordering is stabilized only by the interatomic exchange interaction (independently of DOS used).

Let us investigate the effect of non-interacting DOS form on the magnetization at non-zero temperature. In the case $n<1$, the concentration of electrons with spin projections $\uparrow$ and $\downarrow$ at arbitrary $\operatorname{DOS} \rho(\epsilon)$ and temperature $\Theta$ is as follows:

$$
n_{\uparrow}=\left(1-n_{\downarrow}\right) \int_{-w(n)}^{w(n)} \frac{\rho(t) \mathrm{d} t}{\exp \left(\frac{E_{\uparrow}(t)}{\Theta}\right)+1}, \quad n_{\downarrow}=\left(1-n_{\uparrow}\right) \int_{-w(n)}^{w(n)} \frac{\rho(t) \mathrm{d} t}{\exp \left(\frac{E_{\downarrow}(t)}{\Theta}\right)+1} .
$$

Taking into account $E_{s}(t)=\mu_{s}+\alpha_{s} t$, where $\mu_{s}=-\mu+\beta_{s}-z J_{\text {eff }} n_{s}$, we write (20) as

$$
\frac{n+m}{2-n+m}=\int_{-w(n)}^{w(n)} \frac{\rho(t) \mathrm{d} t}{\exp \left(\frac{\mu_{\uparrow}+\alpha_{\uparrow} t}{\Theta}\right)+1}, \quad \frac{n-m}{2-n-m}=\int_{-w(n)}^{w(n)} \frac{\rho(t) \mathrm{d} t}{\exp \left(\frac{\mu_{\downarrow}+\alpha_{\downarrow} t}{\Theta}\right)+1}
$$

The difference between the values of the shifted chemical potentials for the electrons with different spin projection is:

$$
\mu_{\uparrow}-\mu_{\downarrow}=\beta_{\uparrow}-\beta_{\downarrow}-z J_{\mathrm{eff}} m
$$

The numerical self-consistent analysis of the system of equations (21) and condition (22) has been carried out. We have obtained the magnetization as a function of temperature, electron concentration and energy parameters of the model, exchange interaction and correlated hopping, in particular.

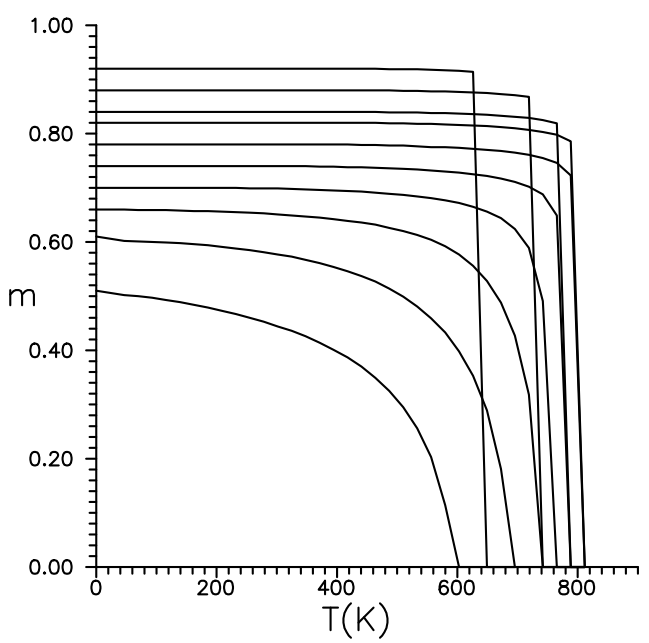

Figure 3. Temperature dependencies of magnetization for the DOS of bcc-lattice at $z J_{\text {eff }} / w=0.08$. The curves (from down to up) correspond to the case of electron concentrations $n=0.58,0.62,0.66,0.70,0.74,0.78,0.82$, $0.84,0.88,0.92$.

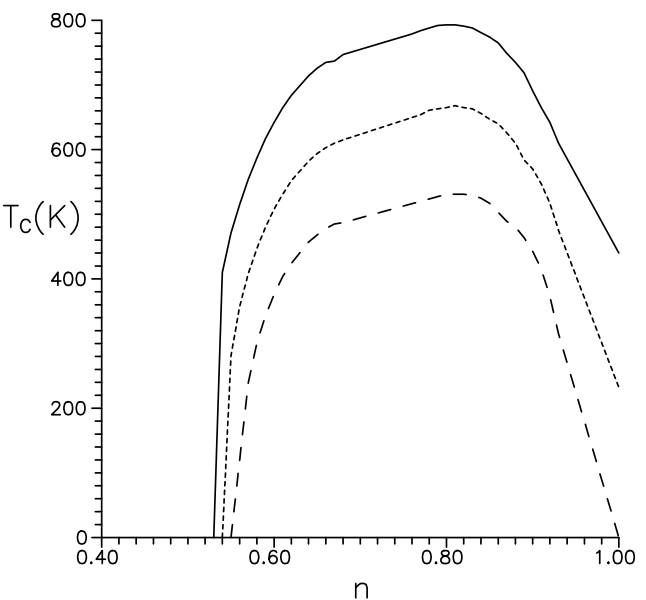

Figure 4. Concentration dependencies of Curie temperature for the DOS of bcc-lattice at $\tau_{1}=0$. The curves (from up to down) correspond to the case of $z J_{\mathrm{eff}} / w=0,0.04$ and 0.08 .

In the case of semi-elliptical DOS, the temperature dependence of magnetization is step-wise and Curie temperature has rather low values. This fact is in accord with concentration dependence of energy difference of paramagnetic and ferromagnetic states $\Delta E_{0}^{\mathrm{FM}} / w$ for the semi-elliptic DOS. With an increase of effective exchange interaction, the Curie temperature increases. In the case of 
DOS with a peak near the band-edge $(a \neq 0)$, the region of ferromagnetic ordering is shifted to low electron concentration values and the difference $\Delta E_{0}^{\mathrm{FM}} / w$ increases, which leads to a substantial increase of Curie temperature.

In the case of DOS, which corresponds to the simple cubic lattice, the dependence $m(\Theta / w)$ is also step-wise; the concentration dependence of Curie temperature has a maximum. Such nonlinear behavior of $T_{\mathrm{C}}(n)$ is also obtained for the rectangular DOS, and Curie temperature is in agreement with experimental values for sulphides of transition metals [44], that have cubic lattice symmetry. Our results for the case of DOS of bcc-lattice are in qualitative agreement with the papers $[40,41,45,46]$, where spectral density approximation (SDA) is used. In figure 3 the temperature dependencies of magnetization at various electron concentrations are plotted (we assume that half-bandwidth $w=1 \mathrm{eV}$, like in the cited works). In the absence of effective exchange interaction we have obtained the values of Curie temperature, which are lower than the results of SDA. We argue that this fact is due to the peculiarities of spin-dependent shift of band centers, which is responsible for the translational mechanism of ferromagnetism stabilization. As one can see from figure 3, when temperature increases at some fixed electron concentration, the continuous (or discontinuous) transition to paramagnetic state is realized. The concentration dependence of Curie temperature (see figure 4) is of nonlinear shape: ferromagnetic ordering occurs at some critical electron concentration (which has been determined based on the ground state energy minimization), $T_{\mathrm{C}}$ increases sharply and at $n \simeq 0.82$ reaches the maximum value. With an increase of effective exchange interaction parameter, the maximum value increases and ferromagnetic concentration region broadens. Since the translational mechanism of ferromagnetism is destabilized by the increase of the correlated hopping parameter $\tau_{1}$, the Curie temperature decreases and the maximum of $\Theta_{\mathrm{C}} / w$ is shifted to the lower electron concentration.

\section{Peculiarities of the electronic conductivity}

Using the method of the papers $[47,48]$, we calculate the $x x$-component of static electronic conductivity in the regime of strong intra-atomic correlation

$$
\sigma=-\sum_{s} \sigma_{0}\left(1-n_{\bar{s}}\right)\left(\int_{-w(n)}^{w(n)} \frac{\rho(t) t \mathrm{~d} t}{\exp \left(\frac{E_{s}(t)}{\Theta}\right)+1}+\int_{-\tilde{w}(n)}^{\tilde{w}(n)} \frac{\rho(t) t \mathrm{~d} t}{\exp \left(\frac{\tilde{E}_{s}(t)}{\Theta}\right)+1}\right),
$$

where the first sum is the conductivity of the lower subband, the second sum is the conductivity of upper subband. Here the magnetization of the system is an important parameter which is calculated using the method of the previous section separately for $n<1$ and $n>1$. In the above equation, $\sigma_{0}$ is a normalizing constant dependent on the mechanism of scattering and lattice parameter. It should be noted here that one should perform a self-consistent calculation of spin-dependent shifts of subband centers which are dependent on the DOS form as well as on band filling and magnetization of the system.

Figure 5 shows the concentration dependencies in Hubbard model (both correlated hopping parameters are taken to be zero) in a strong intraatomic correlation regime. This plot allows us to analyze the effect of translation processes and DOS form on the conductivity of narrowband materials. The dependence of conductivity value on the chosen DOS form is related to the corresponding dependence of kinetic energy. In particular, the maximum value of kinetic energy is reached at rectangular DOS, and the minimum value corresponds to the body-centered cubic DOS. The concentration dependence of conductivity is determined by the kinetic energy of electrons and lattice magnetization. Both these factors, in their turn, depend on the peculiarities of bare density of states and the interatomic interactions, which result in the appearance of correlation narrowing of Hubbard subbands and spin-dependent shifts of subband centers.

The absence of sharp change of conductivity in the vicinity of half subband filling is a characteristic feature of the curve corresponding to the model rectangular DOS. It is caused by the absence of a transition to magnetically ordered state at this form of DOS. The maximum of concentration 


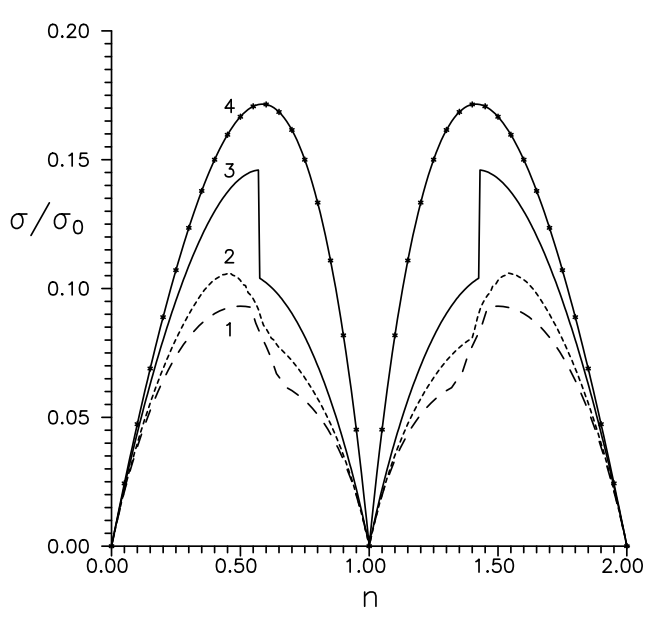

Figure 5. Dependencies of static conductivity on electron concentration at different band DOS forms. Curve 1 corresponds to bcc-lattice, curve 2 corresponds to sc-lattice, curves 3 and 4 are for semi-elliptic and rectangular DOS, respectively.

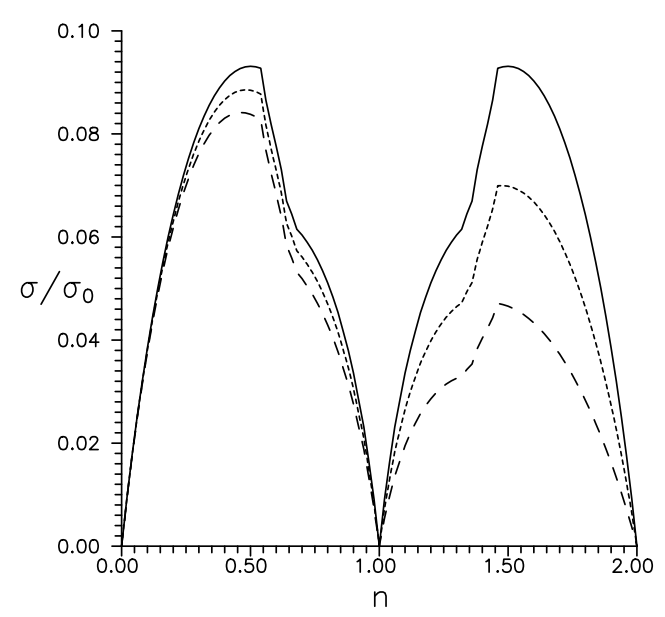

Figure 6. Concentration dependence of conductivity in the system with correlated hopping and bcc-lattice. The correlated hopping parameters for the upper curve are $\tau_{1}=\tau_{2}=$ 0 , for middle curve $\tau_{1}=\tau_{2}=0.1$, for the lower curve $\tau_{1}=\tau_{2}=0.2$.

dependence of $\sigma$ in this case is reached at $n>0.5$, which is typical of paramagnetic state [49]. Stepwise transition to saturated ferromagnetic state which is realized in a system with semi-elliptical DOS, causes a sharp decrease of the conductivity at $n=0.59$. Stabilization of magnetic ordering (with partial spin polarization, see section 4 ), in the systems with body-centered cubic and simple cubic lattices, makes $\sigma(n)$ dependencies more smooth. Note, that in the case of simple cubic lattice, the concentration interval, in which partial magnetic polarization of the system is realized, is much wider than that for the body-centered cubic, which has two important consequences. The first, the changes of conductivity for sc-lattice are more smooth than for bcc-lattice. The second, the difference between maximum values of the conductivity is small, because the maximum of the curve for sc-lattice is in FM region and the respective maximum for bcc-lattice is in PM-region.

In the papers $[48,49]$ the effect of correlated hopping of electrons on the conductivity in the case of rectangular density of states was studied. Equation (23) permits to calculate the concentration dependencies for conductivity of the system with correlated hopping at arbitrary DOS form. As one can see from figures 5 and 6 , both the DOS form and the correlated hopping substantially effect the concentration dependencies of conductivity. Due to correlated hopping, conductivity in more than half-filled band is substantially lower than that in less than half-filled band. An essential peculiarity of the systems with bcc and sc lattices is the decrease of absolute value of conductivity change if the transition takes place in more than half-filled band.

Within the framework of the considered model one can naturally introduce the notions of "wide" (lower) and "narrow" (upper) energy subbands and, correspondingly, "light" and "heavy" current carriers. Using the formula (16) for a coefficient of correlation narrowing of the subband we obtain

$$
m_{\mathrm{eff}}^{s}=\frac{m_{0}}{\left(1-\tau_{1} n\right) \alpha_{s}}
$$

for the effective mass of a current carrier in the lower subband, where $m_{0}$ stands for effective mass of current carriers in the absence of intra-atomic Coulomb correlation and correlated hopping. In a similar way one obtains

$$
\tilde{m}_{\mathrm{eff}}^{s}=\frac{m_{0}}{\left(1-\tau_{1} n-2 \tau_{2}\right) \tilde{\alpha}_{s}}
$$


for carriers in the upper subband. Renormalization of the current carrier mass in the system under consideration is determined by two factors: the correlated hopping of electrons and the correlation narrowing of a band. From the obtained formulae one can see that the effective masses appear to be spin-dependent. Realization of magnetic ordering essentially modifies the behavior of effective masses of current carriers. Conditions for magnetic ordering realization are determined mainly by the form of unperturbed density of electronic states and exchange interaction. Numerical calculations of the magnetization by minimization of the ground state energy permit to study the effect of bare DOS form, model parameters (especially correlated hopping) and external effects (doping, pressure, application of magnetic field) on the behavior of effective masses of current carriers in the lower and upper quasiparticle subbands.

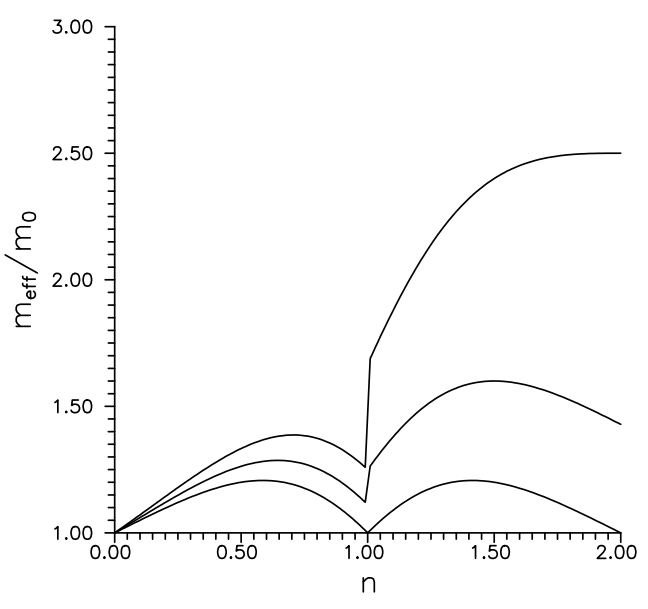

Figure 7. Concentration dependencies of effective mass in paramagnetic state of a system with model rectangular DOS. Curves (from down to up) correspond to values $\tau_{1}=\tau_{2}=0$, 0.1 and 0.2 .

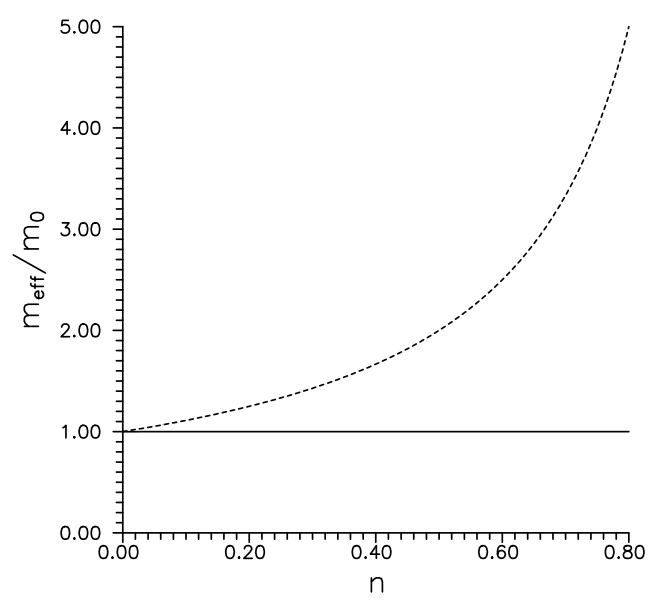

Figure 8. Concentration dependencies of effective masses of carriers with spin $\downarrow$ (upper curve) and $\uparrow$ (lower curve) in a saturated ferromagnetic state $\left(z J_{\text {eff }} / w=0.02, \tau_{1}=0\right)$ for the system with rectangular DOS.

It is worth emphasizing that here the term "effective mass of a current carrier" has a conditional sense [49], which differs to some extent from that used in the standard band theory. Expressions (24) and (25) are related to expressions for the band spectrum, which describe the transitions between $|s\rangle$ - and $|0\rangle$-states and transitions between $|2\rangle$ - i $|s\rangle$-states. For this reason $m_{\text {eff }}^{s}$ and $\tilde{m}_{\text {eff }}^{s}$ are, in fact, effective masses of the corresponding transitions. In the cases, when subbands are almost empty or almost filled, $m_{\text {eff }}^{s}$ and $\tilde{m}_{\text {eff }}^{s}$ can be treated as effective masses of the electron and hole states, respectively (in terms of band theory). In the transition from the regime, in which conduction occurs due to the carriers from the lower band to the state when $s \leftrightarrow 2$-transitions make the main contribution to the current, the effective mass increases stepwise at $n=1$ (qualitatively, behavior of the effective mass on the subband filling is shown in figure 7).

In the considered case, at rectangular density of states, in the absence of an effective exchange interaction, a paramagnetic state is realized in the system [48]. Substantially different behavior is the characteristic feature of the effective mass of carriers at $J_{\text {eff }} \neq 0$ caused by a strong dependence of the energy spectrum on the system magnetization. In figure 8, dependencies of the effective masses of the carriers with different spin directions on the band filling are shown for the case when the magnetization reaches its saturation value. At decreasing $n$, the effective mass of a carrier with the majority spin remains nearly constant, while for the carriers with the minority spin it increases substantially. The localization of carriers with spin $s=\downarrow$ is caused by the peculiarities of the kinetic energy dependence on the system magnetization which makes the motion of the majority spin carriers energetically more favorable. The increase of magnetization leads to the rise of the difference in effective masses of spin-up and spin-down current carriers. This leads to a decrease of overall transport in ferromagnetic state, although effective masses of the carrier with 
the majority spin become lower. Such a dependence of the current carrier mass on projection of spin has been observed recently [50].

\section{Conclusions}

Peculiarities of the modified form of polar model in configurational representation, especially the ability to treat the effects of interatomic Coulomb correlation accurately, permit to illustrate the formation of Hubbard subbands, to incorporate the effects of correlated hopping and to introduce an effective exchange interaction in a natural way. The generalized mean field approximation proposed in the papers [10,16,19], applied to this model, yields physically sound results, temperature and concentration dependent energy spectrum, in particular. It enables us to interpret non-typical temperature transition from metallic state to insulating state observed in the systems $\mathrm{NiS}_{2-x} \mathrm{Se}_{x}$ and $\left(\mathrm{V}_{1-x} \mathrm{Cr}_{x}\right)_{2} \mathrm{O}_{3}$. Using the procedure of numerical self-consistent analysis, temperature dependencies of magnetization have been calculated and the peculiarities of Curie temperature concentration dependence in $\mathrm{Fe}_{1-x} \mathrm{Co}_{x} \mathrm{~S}_{2}$ and $\mathrm{Co}_{1-x} \mathrm{Ni}_{x} \mathrm{~S}_{2}$, electronic conductivity and spin-dependent effective mass of current carriers as a function of energy parameters of the model and electron concentration at various non-perturbed DOS have been investigated. It is worth noting that the application of this approach has allowed to obtain proper estimations for the values of ferromagnetic characteristics for real narrow-band materials.

Thus, the suitability of the model for reasonable description of the peculiarities of electric and magnetic properties of narrow-band material, metal-insulator transition at an increase of temperature, non-typical concentration dependence of Curie temperature and transport properties of Mott-Hubbard materials, in particular, makes the modified form of polar model a convenient and efficient tool for investigation of narrow-band systems.

\section{Acknowledgements}

The authors gratefully acknowledge numerous enlightening discussions with Prof. I.V. Stasyuk.

\section{References}

1. Schubin S., Wonsowsky S., Proc. R. Soc. A, 1934, 145, 159.

2. Hubbard J., Proc. R. Soc. A, 1963, 276, 238.

3. Samsonov V.G., Priadko L.F., Priadko I.F. Electron localization in solids. Nauka, Moscow, 1976 (in Russian).

4. Glauberman A., Vladimirov V., Stasyuk I., Dokl. Akad. Nauk. SSSR, 1959, 126, 543 (in Russian).

5. Glauberman A., Vladimirov V., Stasyuk I., Fiz. Met. Metalloved. [Phys. Met. Metallogr. (USSR)], 1960, 2, 133 (in Russian).

6. Stasyuk I., Visnyk (Ivan Franko National University of Lviv), 1962, 1, 32 (in Ukrainian).

7. Hubbard J., Proc. R. Soc. A, 1965, 285, 542.

8. Didukh L., Stasyuk I., Ukr. Fiz. Zh., 1968, 13, 899 (in Russian).

9. Didukh L., Stasyuk I., Ukr. Fiz. Zh., 1968, 26, 582 (in Russian).

10. Didukh L., J. Phys. Studies, 1997, 1, 241 (in Ukrainian).

11. Kotliar G., Ruckenstein A., Phys. Rev. Lett., 1986, 57, 1362.

12. Zou Z., Anderson P.W., Phys. Rev. B, 1988, 37, 625.

13. Izyumov Yu.A., Usp. Fiz. Nauk [Sov. Phys. - Usp.], 1995, 165, 403 (in Russian).

14. Didukh L., Condens. Matter Phys., 1998, 1, 125.

15. Izyumov Yu.A., Usp. Fiz. Nauk [Sov. Phys. - Usp.], 1997, 167, 465 (in Russian).

16. Didukh L., Phys. Status Solidi B, 1998, 39, R5.

17. Stasyuk I.V., Condens. Matter Phys., 2000, 3, 437.

18. Shvaika A.M., Phys. Rev. B, 2000, 62, 2358.

19. Didukh L., Acta Phys. Pol. B, 2000, 31, 3097.

20. Stasyuk I.V., Shvaika A.M., Ukr. Fiz. Zh., 2002, 47, 975.

21. Stasyuk I.V., Hera O.B. Preprint arXiv:cond-mat/0504289, 2005.

22. Stasyuk I.V., Hera O.B., Condens. Matter Phys., 2006, 9, 587. 
23. Didukh L.D., Stasyuk I.V., Fiz. Met. Metallov., 1972, 33, 429 (in Russian).

24. Harris A.B., Lange R.V., Phys. Rev., 1967, 157, 295.

25. Chao K.A., Spałek J., Oles A., Phys. Status Solidi B, 1977, 84, 747; Chao K.A., Spałek J., Oles A., J. Phys. C., 1977, 10, L271.

26. Mott N.F. Metal-insulator transition. Taylor \& Francis, London, 1990.

27. Yao X., Honig J.M., Hogan T., Kannewurf C., Spałek J., Phys. Rev. B, 1996, 54, 17469; Yao X., Kuo Y.K., Powell D.K., Brill J.W., Honig J.M., Phys. Rev. B, 1997, 56, 7129.

28. Edvards P.P., Rao C.N.R. Metal-Insulator Transitions Revisited. Taylor \& Francis, London, 1995.

29. Arrachea L., Aligia A.A., Phys. Rev. Lett., 1994, 73, 2240.

30. Georges A., Kotliar G., Krauth W., Rozenberg M., Rev. Mod. Phys., 1996, 68, 13.

31. Marra S., Mancini F., Fllega A.M., Matsumoto H., Physica C, 1994, 235-240, 2253.

32. Shvaika A.M., Phys. Rev. B, 2003, 67, 075101.

33. Yuhuan Wen, Yue Yu, Phys. Rev. B, 2005, 72, 045130.

34. Didukh L., Kramar. O., Condens. Matter Phys., 2005, 8, 547.

35. Didukh L., Kramar O., Skorenkyy Yu. Metallic ferromagnetism in generalized Hubbard model. New Developments in Ferromagnetism Reasearch. Ed. V.N. Murray, Nova Science Publishers, Inc., 2006, 39-78.

36. Roth L., Phys. Rev., 1969, 184, 451.

37. Tonegava T., Progr. Theor. Phys., 1974, 56, 1293.

38. Jelitto R., J. Phys. Chem. Solids, 1969, 30, 609.

39. Vollhardt D. Blümer N., Held K., Kollar M., Schlipf J., Ulmke M., Z. Phys. B., 1997, 103, 283.

40. Gaipel G., Nolting W., Phys. Rev. B, 1988, 38, 2608.

41. Hermann T., Nolting W., Solid State Commun., 1997, 103, 351.

42. Shastry B.S., Krishnamurthy B.S., Anderson P.W., Phys. Rev. B, 1990, 41, 2375.

43. Zarubin A.V., Irkhin V.Yu., Fiz. Tverd. Tela, 1999, 41, 1057 (in Russian).

44. Jarrett H.S., Cloud W.H., Bouchard R.J., Butler S.R., Frederick C.G., Gillson J.L., Phys. Rev. Lett., 1968, 21, 617.

45. Hermann T., Nolting W., J. Magn. Magn. Mater., 1997, 170, 253.

46. Nolting W., Potthoff M., Herrmann T., Wegner T. Metallic ferromagnetism - an electronic correlation phenomenon. - In: Band-ferromagnetism. Ground state and finite-temperature phenomena, ed. Baberschke K., Donath M., Nolting W. Springer, Berlin, 2001.

47. Bari R.H., Adler D., Lange R.V., Phys. Rev. B., 1970, 2, 2898.

48. Didukh L., Kramar O., Skorenkyy Yu., Dovhopyaty Yu., Condens. Matter Phys., 2005, 8, 825.

49. Didukh L. Preprint of Institute for Condensed Matter Physics ICMP-03-31U, 2003.

50. McCollam A., Dao R., Julian S.R., Bergeman C., Flouquet J., Aoki D., Physica B, 2005, 359-361, 1.

\title{
Міжелектронні взаємодії у вузьких енергетичних зонах: метод полярної моделі
}

\author{
Л.Д.Дідух, Ю.Л.Скоренький, О.І.Крамар \\ Тернопільський державний технічний університет, Україна, Тернопіль 46001, вул. Руська, 56
}

Отримано 29 квітня 2008 р., в остаточному вигляді - 2 липня 2008 р.

\begin{abstract}
Електронні взаємодії у вузькозонних матеріалах досліджено в рамках модифікованої форми полярної моделі. Ця модель дозволяє врахувати вплив сильної кулонівської кореляції, міжатомного обміну та корельованого переносу електронів і пояснити деякі особливості властивостей вузькозонних матеріалів, зокрема перехід метал-діелектрик при зростанні температури, нелінійну концентраційну залежність температури Кюрі та особливості транспортних властивостей електронної підсистеми. 3 використанням варіанту узагальненого наближення Гартрі-Фока отримано одноелектронну функцію Гріна та квазічастинковий енергетичний спектр моделі. В роботі досліджено перехід металдіелектрик при зміні температури в системі з корельованим переносом. Також досліджено процеси стабілізації феромагнітного впорядкування в системі при різних формах електронної густини станів. Розраховано статичну провідність та спін-залежні ефективні маси носіїв як функції електронної концентрації при різних густинах станів. Показано, що корельований перенос зумовлює асиметрію транспортних та феромагнітних властивостей вузькозонних матеріалів.
\end{abstract}

Ключові слова: полярна модель кристалу, мотт-габбардівський матеріал, феромагнітне впорядкування, провідність

PACS: 71.10.Fd, 71.27.+a, 72.20.-i, 75.10.-b 